\title{
Evaluation of attempted-suicide manage- ment in a rural district of KwaZulu-Natal
}

D C T Nakin, MB ChB, MFamMed

$G$ Joubert, BA, BSC

P J Pretorius, MB ChB, MMed (Psych)

M J V van Vuuren, MB ChB, MPraxMed, PhD

Departments of Family Medicine, Biostatistics and Psychiatry, University of the Free State, Bloemfontein

Objectives. Critical aspects in the management of attempted-suicide patients have been identified in the literature. The aim of this study was to determine which of these aspects were included in the management of patients who attempted suicide in a rural district of KwaZulu-Natal.

Design. Descriptive study.

Setting. The two hospitals in a rural district of KwaZuluNatal.

Subjects. Attempted-suicide patients admitted to the above two hospitals between 1 June 2001 and 31 March 2002.

Outcome measures. Patients were interviewed regarding how they were managed and were followed up until 3 months after the attempted suicide.

Results. Medicine was given to $41 \%$ of participants on admission and $64 \%$ were counselled by a doctor. A nosuicide contract was entered into in $42 \%$ of counselled cases. Family therapy, social worker involvement and psychiatric referral were very low and there was no involvement of friends, teachers or priests.

Conclusion. Management of patients who attempted suicide in this district is inadequate as there are no proper policy guidelines. It is important that health caregivers be trained in psychiatry.

The number of suicide attempts rather than completed suicides has increased, mostly in young people under 25 years of age. ' Correct management of attempted suicide is of crucial importance as mismanagement can fail to prevent further attempts, which could eventually result in completed suicide. Lack of proper management protocols could potentially contribute to improper evaluation of these patients.
The following aspects are critical in the management of attempted-suicide patients. Prompt physical assessment and physical examination must be done to prevent life-threatening complications. The presence of medical conditions causing psychiatric symptoms that might have prompted the person to attempt suicide must also be excluded. Some medical conditions are associated with an increased risk of suicide. ${ }^{2}$ Prompt psychiatric assessment needs to be done immediately the person regains consciousness. This will help to determine whether any psychiatric illness is present and to establish whether the person is on any psychotropic medication. ${ }^{3}$

Family intervention is necessary since lack of communication and problem-solving mechanisms in the family may precipitate a suicide attempt.' Good family support is one of the major factors reducing risk of suicide. Wassenaar ${ }^{4}$ found that over a 3-year period following family therapy there were no hospital readmissions of patients who attempted suicide, while in the group where there was no family intervention the readmission rate was higher.

Implementation of a follow-up system for attempted-suicide patients reduces the repeat rate..$^{5}$ Individual interviews with attempted-suicide patients have been shown to be vital in developing a good relationship and trust between the doctor and the patient. ${ }^{6}$ A never-again contract between the attempted-suicide patient, his/her family and the doctor has also been shown to decrease the repeat rate.

The attitude of health personnel towards the attemptedsuicide patient is important. A negative attitude does not help the patient, and a caring empathic attitude is of vital importance. ${ }^{8}$

Involvement of the social support system (social workers, priests, teachers and friends) in managing the patient greatly reduces the likelihood of repeated attempts.

The rural district under study in KwaZulu-Natal has two hospitals. Available resources for the management of attempted-suicide patients included doctors, psychiatric nurses, social workers and priests, and referral to a psychiatrist or a psychologist. 
The aim of the study was to determine which of the abovementioned aspects were included in management of this group of patients in the district.

\section{Methods}

This descriptive study included attempted-suicide patients admitted to the two hospitals from 1 June 2001 to 31 March 2002. The first author (DCTN) conducted interviews with each participant at their homes or workplaces at least 3 months after admission. The questionnaire was in English. The interviewer is fluent in Xhosa and Sesotho and could easily translate the questionnaire into these languages. The study was retrospective and looked at how these patients were managed. Patients were questioned on how they were managed and were followed up until 3 months after the suicide attempt. In cases where the patient made more than one suicide attempt during the study period, the questionnaire applied to the last attempt.

A pilot study was conducted with 6 people, 3 from each hospital, who had been admitted after attempted suicide.

The protocol was approved by the Ethics Committee of the Faculty of Health Sciences, University of the Free State, and permission to conduct the study was obtained from the two hospitals. Informed written consent was obtained from each participant after a thorough explanation of the study.

\section{Results}

Of the 73 patients admitted during the study period, 59 $(81 \%)$ were interviewed, these being the ones who could be traced and who gave consent. Table I indicates that $56 \%$ of participants were in the 20 -40-year age group, 63\% were female and $85 \%$ were black. The majority of participants (63\%) had some secondary education.

For $88 \%$ of participants this was their first attempt, for $10 \%$ the second attempt and for $2 \%$ their third attempt. Four of the 7 patients who had attempted suicide previously had received treatment. The majority claimed not to have been intoxicated during the suicide attempt (Table II). The majority of the attempts were in December (15\%) and January (15\%). Nearly two-thirds $(64 \%)$ of participants did not lose consciousness. The majority used household poison or prescribed medicine.

Medicines were given to $41 \%$ of the participants on admission and $64 \%$ were counselled by the doctor (Table III). Of the counselled patients, 37\% received a psychiatric assessment and a no-suicide contract was entered into in $42 \%$ of cases. Family intervention was instituted in only $5 \%$ of cases, and

\begin{tabular}{lc}
\multicolumn{2}{l}{ Table I. Demographic characteristics of respondents $(\boldsymbol{N} \mathbf{5 9})$} \\
\hline $\begin{array}{l}\text { Demographic } \\
\text { variable }\end{array}$ \\
\hline Age (yrs) \\
$<20$ \\
$20-40$ & 37.3 \\
$41-60$ & 55.9 \\
Gender & 6.8 \\
Male & \\
Female & 37.3 \\
Race & 62.7 \\
Black & \\
Coloured & 84.5 \\
White & 15.5 \\
Education & 0 \\
$<$ Std 5 & \\
Std 6-9 & 8.5 \\
Std 10 & 62.7 \\
\hline
\end{tabular}

Table II. Present suicide attempt history $(N=59)$

\begin{tabular}{ll}
\hline & $\%$ \\
\hline Communicated suicide intentions beforehand & 17 \\
Intake of drugs before attempt & 28.8 \\
Intoxication during the attempt ( $N=58)$ & 17.2 \\
Method used & \\
Over-the-counter medicine & 1.7 \\
Household poison & 49.2 \\
Prescribed medicine & 44.1 \\
Hanging & 1.7 \\
Other & 3.4 \\
Loss of consciousness & \\
Yes & 32.2 \\
No & 64.4 \\
Do not remember & 3.4 \\
\hline
\end{tabular}

there was no involvement of friends, priest or school teacher. The social worker was involved in 3\% of cases. Only $3 \%$ of cases were told to come back to the hospital for followup, and $19 \%$ of the participants were given medicines to take home. Of these, $46 \%$ indicated that the purpose of prescribing these medicines was not explained. Only 1 patient $(2 \%)$ was referred to a psychiatrist.

No patients were followed up at home by a doctor or a nurse following discharge. Only $3 \%$ were visited by the social worker and 7\% were visited by a priest (Table IV).

Two-thirds (69\%) of the patients felt that the attitude of hospital staff was sympathetic, whereas $27 \%$ felt that they were arrogant. Sixty-nine per cent of patients said that they were 
Table III. Management in hospital $(N=59)(\%)$

\begin{tabular}{|c|c|c|c|}
\hline & Yes & No & Do not remember \\
\hline Medicines given & 40.7 & 25.4 & 33.9 \\
\hline Counselling by doctor & 64.4 & 23.7 & 11.9 \\
\hline Examination by doctor & 49.2 & 35.6 & 15.3 \\
\hline Psychiatric assessment* ( $N=38)$ & 36.8 & 44.7 & 18.4 \\
\hline No-suicide contract* $(N=38)$ & 42.1 & 47.4 & 10.5 \\
\hline Family therapy & 5.2 & 94.8 & \\
\hline Involvement of friends & 0 & 100 & \\
\hline Priest involvement & 0 & 100 & \\
\hline Social worker & 3.4 & 96.6 & \\
\hline School teacher $(N=29)$ & 0 & 100 & \\
\hline Told to come back for review & 3.4 & 96.6 & \\
\hline Given medicine to take at home & 18.6 & 81.4 & \\
\hline Referred to psychiatrist/psychologist & 1.7 & 98.3 & \\
\hline
\end{tabular}

Table IV. Management after discharge from hospital $(N=59)(\%)$

\begin{tabular}{lll}
\hline & Yes & No \\
\hline Contract from hospital & 0 & 100 \\
Visit by doctor & 0 & 100 \\
Visit by priest & 6.8 & 93.2 \\
Visit by social worker & 3.4 & 96.6 \\
Visit by nurse & 0 & 100 \\
\hline
\end{tabular}

happy with the hospital treatment. The majority (81\%) of the patients said that they would not think of committing suicide again, whereas $3 \%$ said that they would consider it and 15\% were uncertain.

\section{Discussion}

The district under study is predominantly rural and there is poor infrastructure with regard to roads and telephones. It was often difficult to locate participants. Because of the vastness of the area the interviewer often had to travel $200 \mathrm{~km}$, only to find that the participant was not at home. Despite these logistical problems a response rate of $81 \%$ was achieved.

The patients were predominantly adolescents or young adults. This is in keeping with the findings of other studies.' The high prevalence of females in this study is also in keeping with previous studies. 1,9 Only 2 white patients were admitted to the two hospitals during the study period, and neither was traceable for interview.
The majority of participants had high school education, which may indicate high levels of stress in this group. The reason for this is unclear but possibly results from unemployment, HIV/ AIDS, marital and other socio-economic problems. Most of the participants were first-time attempters, which may indicate a low repeat rate.

Use of household poison and prescribed medicine by the majority of the participants shows that ingestion was the most convenient way of committing suicide. This has also been demonstrated by other studies. ${ }^{4,9}$ Prescribed medicines and household poison are readily available, therefore care must be exercised to store these appropriately.

A small percentage of the participants were intoxicated during the suicide attempt. This is in contrast with one previous study ${ }^{10}$ which found that $75 \%$ of the participants were intoxicated during the suicide attempt. An overwhelming majority of the participants did not communicate their intentions to commit suicide, which shows that patients are not open about their intentions.

Most attempts were carried out during the holiday months, i.e. December and January. This could point to the fact that people are mostly at home during this period and are not occupied with work or studies. It is easy to think about suicide when one does not have much to do.

The majority of subjects did not lose consciousness, which could indicate that they deliberately acted in such a way as 
to ensure that they would not be successful in their attempt. Studies have shown that parasuicides use more passive methods so as to be discovered and rescued. ${ }^{9}$

Management at these two hospitals revealed that emergency drug treatment is not given to all patients, which could easily lead to loss of life. Counselling and examination by a doctor is not done on all patients, which could lead to improper assessment as organic pathology that might have contributed to the suicide will be missed without examination. Psychiatric assessment is also not done routinely. This will also lead to improper management as psychiatric ailments that might contribute to suicide will be missed. There was no utilisation of the social support system in managing attempted-suicide patients. Social workers, family, priests, friends and school teachers were not involved, which is not in keeping with international norms. Virtually no patients were asked to come back for review and no follow-up mechanism was instituted. This can lead to repeat attempts and eventually lead to complete suicide. The available referral system to a psychiatrist or a psychologist was not utilised.

In conclusion, management of attempted-suicide patients in this district is inadequate as there are no proper policy guidelines. International guidelines for managing attempted suicide are not being followed. It is important that health caregivers be trained, especially in psychiatry. Psychiatrists and psychologists need to visit the hospitals to capacitate health caregivers. Further research is necessary to determine the suicide repeat rate, so as to establish whether improper management contributes to a high repeat rate. It would be interesting to establish how attempted suicide is managed in other hospitals thoughout South Africa.

\section{References}

1. Mhlongo T, Peltzer K. Parasuicide among youth in a general hospital in South Africa Curationis 1999: 22: 72-76

2. Barraclough B. Medical disorders as precursors of suicide. Psychiatry in Practice 1997; 4(2): 7-9.

3. Whiffeld W, Southern DA. Prevention of suicide; some practical steps. I Roy Soc Health 1996: 116: $295-298$

4. Wassenaar DR. Brief strategic family therapy and the management of adolescent Indian parasuicide patients in the general hospital setting. South African Journal of Psychology 1987; 17: 93-99.

5. Dieserud G, Loeb M, Ekeberg $O$. Suicidal behaviour in the Municipality of Baerum, Norway. Suicide Life Threat Behav 2000; 30 (1): 61-73.

6. Pillay AL, Wassenaar DR. Family dynamics, hopelessness and psychiatric disturbance in parasuicidal adolescents. Aust N ZJ Psychiatry 1997; 31: 227-231.

7. Brent DA. The aftercare of adolescents with deliberate self-harm. J Child Psycho Psychiatry 1997; 38: 277-286.

8. Press BR, Khan SA. Management of the suicidal child or adolescent in the emergency department. Curr Opin Pediatr 1997; 9: 237-241.

9. Pillay AL, Schlebusch L. Parasuicide among Indian adolescents. South African Journal of Psychology 1987; 17: 107-1 10.

10. Alem A, Kebede D, Jacobsson L, Kullgren G. Suicide attempts among adults in Butajira Ehiopia. South African Journal of Psychiatry 1998; 88: 1200. 